Göttig: Beitr. z. Kenntn. d. wasserh. Natriumsulfide. 229 dampft das Filtrat auf ein beträchtlich kleineres Volumen ein, setzt Ammoniak und Schwefelammonium und nach dem Erkalten sehr reichlich Schwefelsäure zu und vervollständigt schliesslich die Ausfällung des Germaniumsulfides durch Einleiten von Schwefelwasserstoff, worauf man dieses in bereits beschriebener Weise in wägbares Oxyd überführt.

Freiberg, Laborat. d. Königl. Bergakademie, Juli 1886.

\title{
Beiträge zur Kenntniss der wasserhaltigen Natriumsulfide;
}

\author{
yon
}

\section{Christian Göttig.}

Das Natriummonosulfid wurde bisher für analytisch chemische Zwecke meistens nach Vauquelin und Berzelius durch Vermischen einer mässig concentrirten wässrigen Natronhydratlösung mit der gleichen Menge einer mit Schwefelwasserstoff vollständig gesättigten . Natronlösung von gleichem specifischem Gewicht und darauf folgende Concentration des Gemenges durch Abdampfen im Vacuum als Hydrat von der Zusammensetzung $\mathrm{Na}_{2} \mathrm{~S}+9 \mathrm{H}_{2} \mathrm{O}$ dargestellt. Bei Anwendung einer Natronlauge von sehr hohem specifschem Gewicht beobachtete Finger ${ }^{1}$ ) auch ohne Concentration im Vacuum bei partieller Sättigung mit Schwefelwasserstoff eine krystallinische Ausscheidung von wasserhaltigem Natriumsulfid. Böttger ${ }^{2}$ ) benutzte eine alkoholische

1) Ann. Phys. 128, 635.

2) Ann. Chem. 223, 335. 
Natronhydratlösung zur Darstellung des Schwefelnatriums und erhielt durch vollständiges Sättigen derselben mit Schwefelwasserstoff und Hinzufügen des gleichen Volumens der nicht mit Schwefelwasserstoff behandelten alkoholischen Natronhydratlösung die Verbindung $\mathrm{Na}_{2} \mathrm{~S}+5 \mathrm{H}_{2} \mathrm{O}$.

Durch nachstehend beschriebene Versuche habe ich gezeigt, dass bei theilweiser Sättigung einer alkoholischen Natronlösung mit Schwefelwasserstoff unter geeigneten, näher ermittelten Umständen, eine äusserst reichliche Abscheidung von Krystallen entsteht, deren Zusammensetzung unter ungleichen Bedingungen den vier verschiedenen Formeln: $\mathrm{Na}_{2} \mathrm{~S}+5 \mathrm{H}_{2} \mathrm{O} ; 2 \mathrm{Na}_{2} \mathrm{~S}+11 \mathrm{H}_{2} \mathrm{O} ; \mathrm{Na}_{2} \mathrm{~S}+6 \mathrm{H}_{2} \mathrm{O}$ und $\mathrm{Na}_{2} \mathrm{~S}+9 \mathrm{H}_{2} \mathrm{O}$ entsprechen kann und die bei weiterem Einleiten von $\mathrm{H}_{2} \mathrm{~S}$ wieder verschwinden.

$\mathrm{Zu}$ den bezüglichen Versuchen wurden alkoholische Natronlösungen von etwas verschiedener Concentration, die auch aus Alkohol von ungleichem Procentgehalt bereitet waren, meistens mit gleichem äusserem Erfolge verwendet, in einzelnen Fällen wurde jedoch Schwefelwasserstoff eingeleitet, auch obne dass sich eine vorübergehende Ausscheidung von Krystallen zu erkennen gab.

So exzielte ich z. B. bei Anwendung einer Lösung, die ans etwa 99,8 procentigem Alkohol durch zweistündige Berührung mit Natronhydrat dargestellt war, während des Einleitens von Schwefelwasserstoff keine Krystallbildung, wohl aber eine nachträgliche Ausscheidung sehr feiner Krystallaggregate. - Dasselbe negative Resultat zeigte sich beim Einleiten von Schwefelwasserstoff in 75 procentigen Alkohol, welcher $1 / 2$ Stunde mit Natronhydrat in Berührung war und dann filtrirt wurde wie auch bei Verwendung einer Natronhydratlösung in etwa 65 procentigem Alkohol vom specifischen Gewicht 0,920. Das specifische Gewicht der beiden zuerst genannten Lösungen hatte ich nicht festgestellt, jedenfalls geht hieraus aber hervor, dass der Wassergehalt des verwendeten Alkohols einerseits und der Gehalt der Lösung an Natronhydrat anderntheils innerhalb gewisser Grenzen liegen muss, wenn sich die Krystalle bilden sollen.

Stets günstige Resultate ergeben sich jedoch bei Ver- 
wendung von alkoholischen Natronhydratlösungen, zu deren Bereitung 96- und 96,8 procentiger Alkohol verwendet wurde und deren specifisches Gewicht etwa zwischen 0,900 und 0,933 lag. Es entstanden immer nach kurz dauerndem Einleiten von Schwefelwasserstoff so reichliche Ausscheidungen meist seideglänzender kleiner Krystallnadeln, dass die ganze Lösung zu einem Brei erstarrte. Dasselbe Resultat wurde unter Anderem erzielt bei Verwendung von etwa 75 procent. Alkohol, wenn das specifische Gewicht über 0,990 lag, sowie von ca. $99 \%$ Alkohol, wenn dasselbe zwischen 0,908 und 0,910 variirte.

Bei der Analyse dieser auf angegebene Weise gewonnenen Schwefelverbindungen ergaben sich anfangs ziemlich verschiedene Resultate, welche mitunter weder unter sich zusammenstimmten, noch auch im Einzelnen mit einer vorauszusehenden Formel genau in Einklang zu bringen waren. Als Grund für diese Erscheinung ergab sich bei der einschlägigen Untersuchung hauptsächlich der Umstand, dass die aus einer und derselben ursprünglichen Lösung gewonnenen Krystalle meistens aus zwei verschiedenen Hydraten bestehen, was durch fractionirte Fällung und Analyse der einzelnen abfiltrirten Portionen ermittelt wurde, ferner die Unbeständigkeit des Stoffs in getrocknetem Zustande, die Thatsache, dass durch die verschiedensten Einflüsse schon in sehr kurzer Zeit wesentliche Veränderungen in der Zusammensetzung wahrzunehmen waren.

Im Exsiccator über Chlorcalcium gab der getrocknete Krystallbrei Wasser ab wie nachstehend beschrieben:

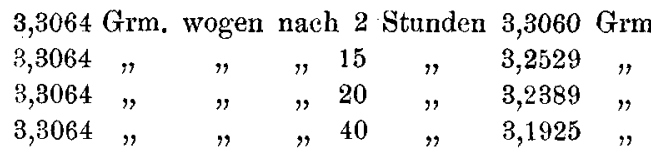

Im offenen Gefäss zeigte sich jedoch folgende Gewichtszunahme:

\begin{tabular}{|c|c|c|c|c|c|c|c|}
\hline 0,8751 & Grm. & woge & na & & & & \\
\hline 0,8751 & $"$ & " & $\eta$ & 3 & $"$ & 0,9108 & , \\
\hline 0,8751 & $"$ & $"$ & $"$ & 4 & $"$ & 0,9196 & \\
\hline, 8751 & $"$ & , & $"$ & $5^{1 / 2}$ & $"$ & 0,9317 & \\
\hline
\end{tabular}


Diese Gewichtszunahme war, wie festgestellt wurde, ausser durch angezogenes Wasser, hauptsächlich durch die Bildung von $\mathrm{Na}_{2} \mathrm{~S}_{2} \mathrm{O}_{3}$ und $\mathrm{Na}_{2} \mathrm{SO}_{4}$ begründet:

0,393 Grm. gaben mit Chlorbarium 3 Stunden nach der Bereitung 0,001 Grm. schwefelsaures Barium.

0,406 Grm. gaben 31/2 Stunden nach der Bereitung 0,0015 Grm. schwefelsaures Barium.

Diese Oxydation vollzog sich beim Erhitzen der Substanz an der Luft bedeutend intensiver, so dass der Glühverlust um $9 \%-10 \%$ geringer sich herausstellte, als dem wirklichen Wasser-Gehalt entsprach.

Auch die Kohlensäure zersetzte die Verbindung unter Bildung von kohlensaurem Natrium und Schwefelwasserstoff.

Aus diesen Gründen verwendete ich nach Ausführung vieler Analysen mit verschiedenen Ergebnissen, welche mich zur Ermittelung der vorstehend angegebenen Thatsachen führten, stets ganz frisch bereitete, mit Alkohol sorgfältig ausgewaschene Substanz und untersuchte die zuerst gebildeten Krystalle getrennt von den zuletzt entstandenen. Doch wurden auch hier kleinere Abweichungen beobachtet, die sich allerdings erklären, wenn man sowohl die Methode der Ausführung der Analyse, wie auch die begleitenden Verhältnisse bei den betreffenden Bestimmungen, welche ich im Folgenden zusammengestellt habe, in Betracht zieht.

Die Natriumbestimmungen wurden theils durch Ueberführung in Chlornatrium, längeres Erhitzen in sehr heissem Luftbade und vorsichtiges Erwärmen mit der Bunsen'schen Flamme (ohne Glühen), zum Theil durch Zersetzung mittelst Schwefelsäure ausgeführt. Bei der Zersetzung durch Säuren zeigte sich meistens eine äusserst geringe Trübung durch abgeschiedenen Schwefel.

Die Schwefelbestimmungen wurden durch Ueberführung in schwefelsaures Barium sowie in Schwefelcadmium ${ }^{1}$ ) ausgeführt.

1) Beim Auswaschen dieses Niederschlages gab das zum Waschen benutzte Wasser nach achtstündigem Auswaschen mit Schwefelwasserstoff eine gelbe Färbung; dem entsprechend wurde der Schwefelgehalt zueh höher gefunden. 
Zur Bestimmung des Wassers wurde die Verbindung in Kugelröhren im Luftbade bis über $200^{\circ}$ in der Weise erhitzt, dass vor dem Einfüllen der Substanz alle Luft durch Wasserstoff ersetzt war, und dass dieses Gas während des Einfüllens, Erhitzens und Abkühlens bis zum Verschliessen die Röhre durchströmte. Der wasserfreie Rückstand besass eine hellrothe Farbe.

Nachstehend folgt eine tabellarische Uebersicht der Resultate der Analysen:

Tabelle I enthält die Resultate der Analysen der feinen Krystallnadeln, welche immer zuerst beim Einleiten des Schwefelwasserstoff's sich bilden.

Tabelle II zeigt die Resultate der Analysen der etwas grösseren und festeren, theils nadelförmigen, theils körnigen Krystalle, welche bei der fractionirten Ausfällung mittelst Schwefelwasserstoffs zuletzt gewonnen wurden.

\section{Tabelle I.}

\begin{tabular}{|c|c|c|c|c|c|c|}
\hline & \multicolumn{3}{|c|}{$\begin{array}{l}\text { Gefundene } \\
\text { Werthe in } \\
\text { Procenten für }\end{array}$} & \multirow{2}{*}{\multicolumn{2}{|c|}{\begin{tabular}{|c|} 
Specifisehes \\
Gewicht \\
der ur- \\
des \\
sprüngl. Alko- \\
Lösung
\end{tabular}}} & \multirow[t]{2}{*}{$\begin{array}{l}\text { Bemerkungen über die ein- } \\
\text { zelnen Bestimmungen }\end{array}$} \\
\hline & $\mathrm{Na}$ & $\mathrm{S}$ & $\mathrm{H}_{2} \mathrm{O}$ & & & \\
\hline I & 24,19 & 一 & - & 1,028 & 0,901 & \multirow{7}{*}{$\begin{array}{l}\text { Die Substanzen wurden aus } \\
\text { frisch bereiteter Lauge her- } \\
\text { gestellt, sofort durch Filtra- } \\
\text { tion isolirt, schnell zwischen } \\
\text { Fliesspapier getrocknet und } \\
\text { gleich untersucht. }\end{array}$} \\
\hline II & 24,24 & - & - & 1,028 & 0,901 & \\
\hline III & 24,68 & - & - & 0,910 & 0,795 & \\
\hline IV & 24,20 & - & - & 0,995 & 0,860 & \\
\hline V & 24,16 & - & $\cdots$ & 0,908 & 0,795 & \\
\hline VI & 24,62 & - & 57,8 & 0,922 & 0,809 & \\
\hline VII & 24,63 & 一 & - & 0,922 & 0,809 & \\
\hline VIII & 24,8 & - & 58,48 & 0,922 & 0,809 & \\
\hline IX & 21,5 & - & - & 0,995 & 0,860 & $\left\{\begin{array}{l}\text { Supst. War etwa } \text { stunde vor } \\
\text { d. Filtration in d. Mutterlösung. }\end{array}\right.$ \\
\hline
\end{tabular}

Die Resultate der Tabelle I entsprechen alle der Formel $\mathrm{Na}_{2} \mathrm{~S}+6 \mathrm{H}_{2} \mathrm{O}$, welche verlangt:

$$
\begin{aligned}
\mathrm{Na} & =24,73 \\
\mathrm{~S} & =17,20 \\
\mathrm{H}_{2} \mathrm{O} & =58,06
\end{aligned}
$$


234 Göttig: Beiträge zur Kenntniss der

Tabelle II.

\begin{tabular}{|c|c|c|c|c|c|c|}
\hline \multirow[b]{2}{*}{$=1$} & \multicolumn{3}{|c|}{$\begin{array}{c}\text { Gefundene } \\
\text { Werthe in } \\
\text { Procenten für }\end{array}$} & \multirow{2}{*}{\multicolumn{2}{|c|}{$\begin{array}{c}\text { Specifisches } \\
\text { Gewicht } \\
\text { der ur- } \\
\text { sprüngl. Alk } \\
\text { Löksung } \\
\text { Lösols } \\
\end{array}$}} & \multirow[t]{2}{*}{$\begin{array}{l}\text { Bemerkungen über die ein- } \\
\text { zelnen Bestimmungen }\end{array}$} \\
\hline & $\mathrm{Na}$ & $\mathbf{s}$ & $\mathrm{H}_{2} \mathrm{O}$ & & & \\
\hline $\mathbf{I}$ & - & - & 66,3 & 0,995 & 0,863 & $\left\{\begin{array}{l}\text { Gleich nach der Ausfällung } \\
\text { isolirt }\end{array}\right.$ \\
\hline II & - & - & 67,1 & 0,995 & 0,858 & Substanz stand 18 Stunden \\
\hline III & 18,94 & - & - & 0,995 & 0,858 & der \\
\hline IV & 19,04 & -- & - & 0,995 & 0,858 & \\
\hline $\mathrm{V}$ & 19,2 & -- & - & 0,995 & 0,858 & $\left\{\begin{array}{l}\text { Substanz wurde längere Zeit } \\
\text { m. } 99,8 \% \text { Alkohol gewasch. }\end{array}\right.$ \\
\hline VI & - & - & 57,1 & 0,925 & 0,808 & m. $99,8 \%$ Alkohol gewasch. \\
\hline VII & 26,2 & - & - & 0,915 & 0,808 & \\
\hline VIII & - & 17,86 & 一 & 0,910 & 0,808 & $\mathrm{~S}$ als $\mathrm{BaSO}_{4}$ best. gleich \\
\hline IX & - & 17,80 & - & 0,910 & 0,808 & \begin{tabular}{l|l} 
wie VIII & nach der
\end{tabular} \\
\hline $\mathbf{X}$ & - & 18,47 & - & 0,915 & 0,808 & $\mathrm{~S}$ als CdS best. \\
\hline $\mathrm{XI}$ & - & 18,14 & - & 0,915 & 0,808 & wie $\mathbf{X} \quad$ analysirt \\
\hline $\mathrm{XII}$ & 25,8 & $-\cdots$ & - & 0,930 & 0,808 & \multirow{6}{*}{ 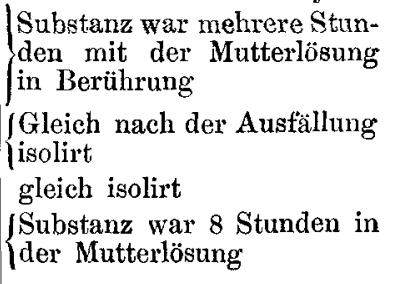 } \\
\hline XIII & 26,3 & - & - & 0,930 & 0,808 & \\
\hline XIV & 26,2 & - & - & 0,930 & 0,808 & \\
\hline $\mathrm{XV}$ & 25,7 & - & - & 0,910 & 0,795 & \\
\hline XVI & 25,6 & - & 56,1 & 0,990 & 0,858 & \\
\hline XVII & 26,65 & - & $\longrightarrow$ & 0,910 & 0,795 & \\
\hline XVIII & 25,45 & - & - & 0,905 & 0,808 & \multirow{13}{*}{$\begin{array}{l}\text { mit Benutzung der Wasser- } \\
\text { luftpumpe sofort nach dem } \\
\text { Entstehen schnell filtrirt, } \\
\text { mit } 96 \% \text { Alkohol ausge- } \\
\text { waschen, in etwa } 20 \text { Minuten } \\
\text { zwischen Fliesspapier ge- } \\
\text { trocknet und gleich unter- } \\
\text { sucht }\end{array}$} \\
\hline $\mathrm{XIX}$ & 25,8 & - & 57,4 & 0,920 & 0,809 & \\
\hline $\mathrm{XX}$ & 25,2 & - & 55,7 & 0,998 & 0,809 & \\
\hline XXI & 25,4 & - & 56,30 & 0,921 & 0,809 & \\
\hline $\mathrm{XXII}$ & - & - & 56,2 & 0,920 & 0,809 & \\
\hline XXIII & 25,66 & - & 55,72 & 0,920 & 0,809 & \\
\hline XXIV & 25,58 & 一 & 57,03 & 0,915 & 0,809 & \\
\hline $\mathrm{XXV}$ & 25,56 & - & - & 0,920 & 0,809 & \\
\hline $\mathrm{XXVI}$ & 25,69 & - & - & 0,920 & 0,809 & \\
\hline XXVII & 25,43 & - & - & 0,920 & 0,809 & \\
\hline XXVIII & 25,29 & - & - & 0,920 & 0,809 & \\
\hline XXIX & 25,85 & - & 56,48 & 0,920 & 0.809 & \\
\hline $\mathbf{X X X}$ & 25,64 & - & $1-$ & 0,920 & 0,809 & \\
\hline
\end{tabular}

Die Resultate der Analysen I-V in Tabelle II stehen im Einklang mit der Verbindung $\mathrm{Na}_{2} \mathrm{~S}+9 \mathrm{H}_{2} \mathrm{O}$ welche enthält: 


$$
\begin{array}{r}
\mathrm{Na}=19,17 \\
\mathrm{~S}=13,33 \\
\mathrm{H}_{2} \mathrm{O}=67,50 \\
\hline 100,00
\end{array}
$$

Die bei den meisten übrigen Analysen der Tabelle II gefundenen Werthe entsprechen im Durchschnitt der Formel $2 \mathrm{Na}_{2} \mathrm{~S}+11 \mathrm{H}_{2} \mathrm{O}$, welche verlangt:

$$
\begin{array}{r}
\mathrm{Na}=25,98 \\
\mathrm{~S}=18,09 \\
\mathrm{H}_{2} \mathrm{O}=55,93 \\
\hline \mathbf{1 0 0 , 0 0}
\end{array}
$$

In einzelnen Fällen nähert sich der gefundene Natriumgehalt dem für die Formel $\mathrm{Na}_{2} \mathrm{~S}+5 \mathrm{H}_{2} \mathrm{O}$ berechneten Werth: $\mathrm{Na}=27,4, \mathrm{~S}=19,0, \mathrm{H}_{2} \mathrm{O}=53,6$.

Aus vorstehenden Resultaten, welche in Tabelle. I und II zusammengestellt sind, schien mir hervorzugehen, dass unter Umständen das specifische Gewicht der alkoholischen Natronlösung und der Procentgehalt des verwendeten Alkohols auf die Zusammensetzung der Sulfurete von Einfluss sein könnte, dass ein längeres Verweilen der Verbindung in einer wasserarmen alkoholischen Mutterlösung den Wassergehalt vermindere, wogegen bei Verwendung von mit Wasser verdünntem Alkohol die längere Berührung mit der Mutterlauge vor der Isolirung eine Vermehrung des Wassergehaltes zur Folge haben müsse.

Um diese Beziehungen mit Sicherheit klar zu legen, wurden folgende Versuche angestellt:

a) Durch partielle Sättigung einer aus etwa 99 procent. Alkohol bereiteten Natronhydratlösung mit Schwefelwasserstoff stellte ich einen Krystallbrei dar, der 28 Tage in der ersten Mutterlösung belassen und dann analysirt wurde. Die Substanz hatte sich nach dieser Zeit in ihrem Habitus nicht sichtbar verändert; die Natriumbestimmung ergab jedoch folgendes Resultat:

1. $0,5407 \mathrm{Grm}$. Substanz gaben $0,3692 \mathrm{NaCl}=26,85 \% \mathrm{Na}$.

2. 0,5038 Grm. Substanz gaben $0,3453 \mathrm{NaCl}=26,96 \% \mathrm{Na}$.

b) Aus 75 procent. Alkohol wurde die Substanz in derselben Weise wie bei a) angegeben dargestellt und nach 30 tägiger Berührung mit der Mutterlösung untersucht. Der 
Habitus hatte sich vollständig verändert, die Krystallform erschien unter dem Mikroskop nicht mehr nadelförmig, sondern octaëdrisch, die Natriumbestimmung ergab folgendes Resultat, das der Formel $\mathrm{Na}_{2} \mathrm{~S}+9 \mathrm{H}_{2} \mathrm{O}$ entspricht:

1. Aus $0,5133 \mathrm{Grm}$. Subst. entstanden $0,2509 \mathrm{Grm}$. $\mathrm{NaCl}=19,20 \% \mathrm{Na}$.

2. Aus 0,4984 Grm. Subst. cntstanden 0,2414 Grm. $\mathrm{NaCl}=19,04 \% \mathrm{Na}$.

Berechn, nach der Formel:

Gcfunden:

$\begin{array}{cccc}\mathrm{Na}_{2} \mathrm{~S}+9 \mathrm{H}_{2} \mathrm{O} & \mathrm{I} & \mathrm{II} . & \text { Mittel } \\ \mathrm{Na}=1 \mathrm{G}, 17 & \mathrm{Na}=19,20 & 19,04 & 19,12 \\ \mathrm{~S}=13,33 & \mathrm{~S}=- & - & - \\ \mathrm{H}_{2} \mathrm{O}=67,50 & \mathrm{H}_{2} \mathrm{O}=- & - & -\end{array}$

c) In derselben Weise wurden die Krystalle aus etwa 96,8 procent. Alkohol dargestellt, mit demselbelben Alkohol gewaschen, isolirt und dann aus etwa 96,8 procent. Alkohol umkrystallisirt. Die Krystalle zeigten eine grössere Beständigkeit, als die des nicht umkrystallisirten Sulfurets; bei der Zersetzung durch Säure ergab sich keine Trübung von ausgeschiedenem Schwefel.

Bei der Analyse wurden folgende Resultate erhalten:

0,8208 Grm. Substanz gaben beim Erhitzen im H 0,4398 Grm. $=53,58 \% \mathrm{H}_{2} \mathrm{O}$ ab.

$0,4808 \mathrm{Grm}$. Substanz lieferten mit Schwefelsäure zersetzt $0,4078 \mathrm{Grm}$. $\mathrm{Na}_{2} \mathrm{SO}_{4}=27,48 \%$ Na.

Berechn. nach der Formel $\mathrm{Na}_{2} \mathrm{~S}+5 \mathrm{H}_{2} \mathrm{O}$ :

$$
\begin{gathered}
\text { Gefunden: } \\
\mathrm{Na}=27,49 \\
\mathrm{~S}=- \\
\mathrm{H}_{2} \mathrm{O}=53,58
\end{gathered}
$$$$
\mathrm{Na}=27,4
$$$$
\mathrm{S}=19,0
$$$$
\mathrm{H}_{2} \mathrm{O}=53,6
$$

d) Die aus 96,8 procent. Alkohol hergestellten Nadeln wurden aus etwa 99,8 procent. Alkohol umkrystallisirt; die Analyse der Krystalle ergab folgendes:

0,7543 Grm. Substanz gaben 0,4033 Grm. $=53,47 \% \mathrm{H}_{2} \mathrm{O}$.

$0,3301 \mathrm{Grm}$. Substana gaben 0,2709\% $\mathrm{Na}_{2} \mathrm{SO}_{4}=26,6 \%$ Na.

Berechn. nach der Formel $\mathrm{Na}_{2} \mathrm{~S}+5 \mathrm{H}_{2} \mathrm{O}$ :

Gefunden:

$$
\begin{array}{rlrl}
\mathrm{Na} & =27,4 & \mathrm{Na} & =26,6 \\
\mathrm{~S} & =19,0 & \mathrm{~S} & =- \\
\mathrm{H}_{2} \mathrm{O} & =53,6 & \mathrm{H}_{2} \mathrm{O} & =53,47
\end{array}
$$

e) Die aus 96,8 procent. Alkohol dargestellte Substanz wurde aus 75 procent. Alkohol umkrystallisirt. Erst nach mehreren Stunden schieden sich büschelförmig strahlige Krystalldrusen aus. - Die Krystalle erschienen unter der Lupe 
sehr schön ausgebildet als Combinationen der quadratischen Säule mit dem Quadratoctaëder derselben Ordnung; die Säulenkanten schienen bei einigen Krystallen durch die Säule zweiter Ordnung abgestumpft. Die Analyse ergab folgendes Resultat:

Aus $0,7921 \mathrm{Grm}$. Substanz wurden $0,3764 \mathrm{Grm} . \mathrm{NaCl}=18,68 \% \mathrm{Na}$ erhalten.

$\begin{array}{rlrl}\text { Berechn. nach der Formel } \mathrm{Na}_{2} \mathrm{~S}+9 \mathrm{H}_{2} \mathrm{O}: & \text { Gefunden: } \\ \mathrm{Na}=19,17 & \mathrm{Na}=18,68 \\ \mathrm{~S}=13,33 & \mathrm{~S}=- \\ \mathrm{H}_{2} \mathrm{O}=67,50 & \mathrm{H}_{2} \mathrm{O}=-\end{array}$

Die Resultate der vorstehend beschriebenen Versuche lassen sich folgendermassen zusammenfassen:

I. Durch Behandeln einer nicht zu verdünnten Lösung von Natronhydrat in hochprocentigem Alkohol mit Schwefelwasserstoff entsteht bei einem gewissen Grade der Sättigung mit diesem Gase zunächst die Verbindung $\mathrm{Na}_{z} \mathrm{~S}$ $+6 \mathrm{H}_{2} \mathrm{O}$, darauf, nachdem die Lösung wasserärmer geworden ist, das Hydrat $2 \mathrm{Na}_{2} \mathrm{~S}+11 \mathrm{H}_{2} \mathrm{O}$ und unter Umständen das Sulfuret $\mathrm{Na}_{2} \mathrm{~S}+5 \mathrm{H}_{2} \mathrm{O}$, welche Stoffe alle bei weiterer Einwirkung des Schwefelwasserstoffs schnell wieder verschwinden. Diese Substanzen verlieren bei Berührung mit annähernd oder ganz wasserfreiem Alkohol allmählich einen Theil ihres Krystallwassers, verwandeln sich dagegen in Alkohol von grösserem Wassergehalt unter Aufnahme von Wasser und Aenderung der Krystallform in die Verbindung $\mathrm{Na}_{2} \mathrm{~S}+9 \mathrm{H}_{2} \mathrm{O}$. Aus hochprocentigem Allkohol umkrystallisirt, entsteht das Sulfuret $\mathrm{Na}_{2} \mathrm{~S}+5 \mathrm{H}_{2} \mathrm{O}$, aus wässerigem Alkohol umkrystallisirt das Hydrat $\mathrm{Na}_{2} \mathrm{~S}+9 \mathrm{H}_{2} \mathrm{O}$.

Il. Durch Einwirkung von Schwefelwasserstoff auf eine concentrirte Lösung von Natronhydrat in wasserreichem Alkohol entsteht zuerst unter dem Einfluss der Zersetzungswärme das Hydrat $\mathrm{Na}_{2} \mathrm{~S}+6 \mathrm{H}_{3} \mathrm{O}$, später die Verbindung $\mathrm{Na}_{2} \mathrm{~S}+9 \mathrm{H}_{2} \mathrm{O}$.

Berlin, im Juli 1886. 